\title{
Treatment of fungal myositis with intra-lesional and intravenous itraconazole: a case report
}

\author{
Xiao-Ji Lin, Rong-Xin Yao ${ }^{*}$, Mu-Qing He, Bao-Ling Zhu and Wen-Jian Guo
}

\begin{abstract}
Introduction: Fungal myositis is very uncommon, even in patients who are immunocompromised. Because of its rarity and a lack of clinical experience, no consensus has been reached about the best means of treating fungal myositis. To the best of our knowledge this is the first description of the treatment of fungal myositis with simultaneous intravenous and intra-lesional itraconazole.
\end{abstract}

Case presentation: A 35-year-old Chinese woman with acute myelomonocytic leukemia developed Candida krusei fungemia and fungal myositis in the right biceps brachii after chemotherapy. A course of intravenous itraconazole and subsequently intravenous voriconazole was initiated and her blood cultures became sterile; however, our patient remained febrile and the myositis did not resolve. Intravenous itraconazole was restarted simultaneously with low-dose intra-lesional itraconazole. The pyrexia settled after 48 hours and within 10 days the lesion could be seen to be resolving. After the course of intravenous and intra-lesional anti-fungals was complete, oral itraconazole was administered as maintenance therapy.

Conclusions: To the best of our knowledge this is the first case in which fungal myositis was successfully treated with intravenous and intra-lesional itraconazole in a patient with acute myelomonocytic leukemia. The efficacy and safety of locally-administered itraconazole to treat intractable soft tissue infections requires further evaluation.

Keywords: Candida krusei, Fungal myositis, Intra-lesional itraconazole

\section{Introduction}

There has been a steady increase in the frequency of opportunistic invasive fungal infections (IFIs) in patients who are immunocompromised, mostly affecting the broncho-pulmonary tract [1]. Even so, fungal myositis, an infection of skeletal muscle, is rare. Regardless, several cases of fungal myositis caused by Candida, Aspergillus and Cryptococcus have been reported in patients who are immunocompromised [2-5]. As it is rare, individual clinicians have rarely seen more than a handful of cases and therefore no evidence-based treatment guidelines have been established beyond the prompt systemic administration of effective anti-fungal agents. We report a case in which fungal myositis was treated effectively with intra-lesional and intravenous itraconazole in a woman with acute myelomonocytic leukemia.

\footnotetext{
* Correspondence: yaorx828@163.com

Department of Haematology, The Second Affiliated Hospital of Wenzhou Medical College, Zhejiang, China
}

\section{Case presentation}

A 35-year-old, $52 \mathrm{~kg}$ Chinese woman with acute myelomonocytic leukemia $\left(\mathrm{M}_{4}\right)$ was admitted to our hospital with fever. Our patient had been diagnosed as having leukemia seven months previously and had received only three cycles of standard induction chemotherapy (cytarabine $100 \mathrm{mg} / \mathrm{m}^{2}$ on days one to seven and with idarubicin $10 \mathrm{mg} / \mathrm{m}^{2}$ on days one to three) due to difficulty in paying for the treatment. At the time of her admission she was initially treated with broad-spectrum antibiotics (cefoperazone-sulbactam and levofloxacin).

The results of a physical examination revealed superficial multiple lymphadenectasis, and our patient's general condition was good. A full blood count revealed a neutrophil count of 940 cells $/ \mathrm{mm}^{3}$. Urine analysis, a computed tomography (CT) scan of the chest and abdominal ultrasonography results were normal. Blood, urine and stool cultures were also negative. Bone marrow cytomorphology showed that monoblasts and promonocytes comprised 48.6 percent of non-erythroid cells. Subsequently, our patient received fludarabine, cytarabine, and granulocyte- 
macrophage colony-stimulating factor (FLAG) chemotherapy (cytarabine $1 \mathrm{~g} / \mathrm{m}^{2}$ on days one to five, with fludarabine $25 \mathrm{mg} / \mathrm{m}^{2}$ also on days one to five). After five days of chemotherapy, the neutrophil count had fallen to 150 cells $/ \mathrm{mm}^{3}$ so she was treated with granulocytemacrophage colony-stimulating factor. At day 7, high pyrexia was evident, but the results of a repeat chest CT scan was normal and blood cultures were sterile. Empiric therapy with imipenem/cilastatin and vancomycin was started. On day 13, high pyrexia recurred and subsequently two separate peripheral venous blood specimens grew Candida krusei, which was sensitive to itraconazole, voriconazole and amphotericin $\mathrm{B}$, but resistant to fluconazole and 5-fluorouracil. Intravenous itraconazole $(400 \mathrm{mg}$ loading dose for the first two days followed by $200 \mathrm{mg}$ daily) was started (Figure 1). On the 15th day our patient reported right upper arm pain with signs of erythema, swelling and hyperalgesia; ultrasonography revealed a hypo-echoic lesion within the right biceps brachii, $19 \times 55 \mathrm{~mm}$ in size, in which blood flow could be detected by color Doppler flow imaging (Figure 2). By day 17, her temperature had gone down to 38.0 to $38.5^{\circ} \mathrm{C}$, but at day 25 a low grade fever persisted and the biceps lesion had not changed, although the blood cultures no longer grew Candida. Histopathological examination of a biopsy specimen from the lesion showed dense fungal spores, no hyphae, but evidence of focal myocyte necrosis and inflammatory cell infiltration (Figure 3a-c). Spore morphology, as demonstrated by periodic acid-Schiff (PAS) and Grocott's methenamine silver (GMS) staining, was characteristic of fungi. The muscle specimen was inoculated on medium and the culture grew C. krusei, confirming the histopathological diagnosis. Anti-fungal susceptibility tests found sensitivities similar to that of the blood culture. Consequently, intravenous itraconazole was substituted with voriconazole (200mg twice daily) for seven days (days 28 to 34 ), as antibiotics were gradually discontinued. However, the pyrexia persisted and the lesion had not diminished in size. Our patient declined the offer of excision, so

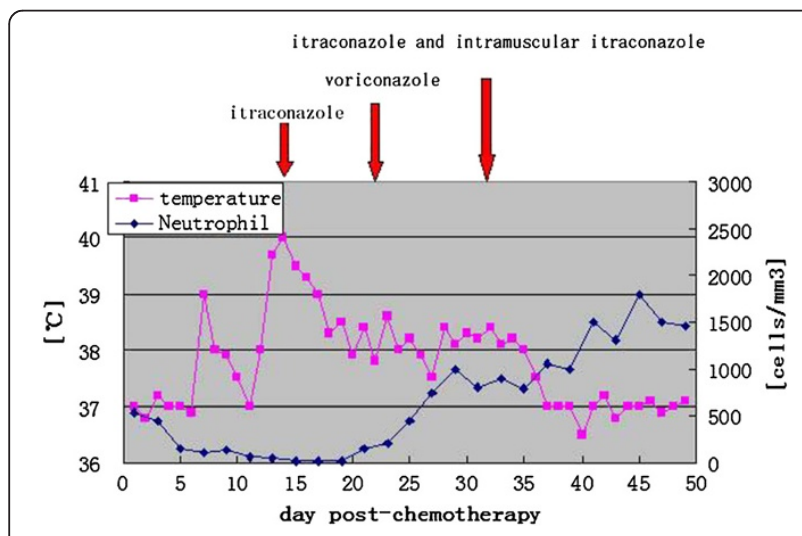

Figure 1 Thermometric evolution and anti-fungal drug therapy.

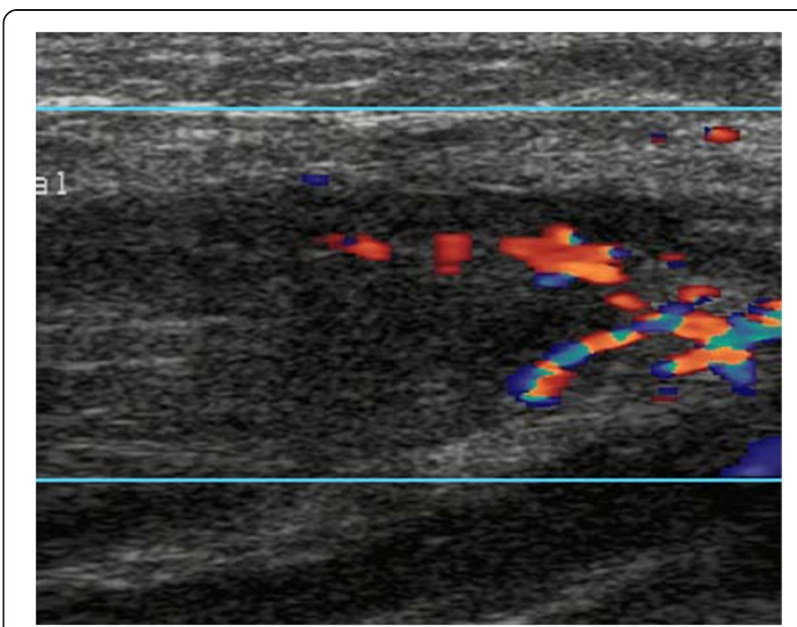

Figure 2 Ultrasonography of the right biceps brachii showed a hypo-echoic lesion within the muscle in which blood supply could be detected by color Doppler flow imaging.

intravenous itraconazole was initiated once again and the decision was made to inject itraconazole $5 \mathrm{mg}$ daily into the lesion. Her fever gradually subsided within 48 hours of commencing intra-lesional itraconazole. Then, 10 days later, a further ultrasound scan demonstrated almost complete resolution of the lesion. Once the neutropenia had resolved, the intravenous and intra-lesional itraconazole were discontinued in favor of oral maintenance therapy with itraconazole 200mg twice daily. A follow-up ultrasound scan one month later showed complete resolution of the muscle lesion.

\section{Discussion}

IFI is a recognized consequence of an immunocompromised state, but there are many possible underlying causes. These include neutropenia, exposure to high-dose corticosteroids, the presence of intravascular catheters and prolonged exposure to broad-spectrum antibiotics. Fludarabine also impairs cell-mediated immunity and can contribute to the initiation of IFIs. To the best of our knowledge this is the first case report of a fungal myositis arising in a patient who was neutropenic with acute myelomonocytic leukemia during fludarabine-based chemotherapy in China. Most infectious myositis is bacterial in etiology, with Staphylococcus aureus being the most common causative organism. Fungal myositis, although rare, has been described and is a recognized complication of systemic mycosis [2,6-8]. The Candida species most often implicated in fungal myositis include Candida tropicalis, C. krusei and Candida albicans [6-8]. The potential routes of infection are generally considered to be hematogenous dissemination, iatrogenic or traumatic with direct inoculation of spores. In our patient's case, as they had no history of trauma or longterm indwelling catheters, the infection is most likely to have resulted from hematogenous dissemination. 

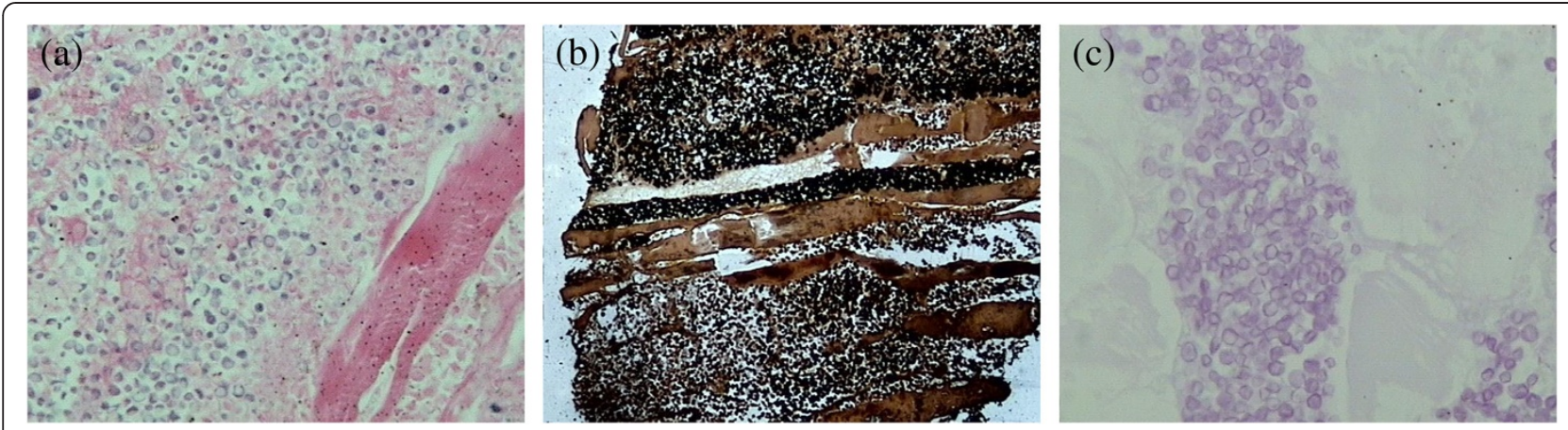

Figure 3 A histopathological analysis of muscle tissue showed focal necrosis with inflammatory cell infiltration and diffuse fungal spores among the muscle cells. (a) hematoxylin and eosin stain, $\times 100$; (b) Grocott's methenamine silver stain, $\times 100$; (c) periodic acid-Schiff stain, $\times 400$.

Apart from the treatment of aspergilloma in fibrocavitary lung disease with intra-cavitary amphotericin B [9-11] and Candida joint infection with intra-articular amphotericin B [12,13], the local administration of antifungal drugs for IFIs is uncommon. Itraconazole was registered for use in 1991 and is widely used to treat several forms of mycosis; however, to the best of our knowledge this is the first case report of the use of intra-lesional itraconazole for fungal myositis.

Pharmacokinetic studies have shown that itraconazole is extensively taken up by the tissues in normal adults and therapeutic levels of itraconazole are maintained many times higher in some infected tissues than plasma. Regardless, in our patient's case she remained febrile and the myositis persisted despite 22 days of intravenous itraconazole and voriconazole. It is not clear why therapeutic levels sufficient to treat the myositis successfully were not attained in our patient's case, although it may be that surrounding ischemic and necrotic muscle reduced tissue penetration. We believe that in our patient's case the systemic treatment alone was inadequate and hypothesize that the use of the combination of intravenous itraconazole and low-dose intra-lesional itraconazole accounted for our patient's clinical improvement. However, the influence of low-dose intra-lesional itraconazole in the successful outcome of our patient's case is not completely clear. The clinical response observed could also have been the result of prolonged administration of intravenous itraconazole in the context of recovering neutropenia and the use of oral maintenance itraconazole therapy. No side effects were observed as a result of the intra-lesional administration of itraconazole.

Our patient's case shows that intra-lesional treatment of fungal myositis with itraconazole is safe and feasible. Future trials should investigate the optimum intralesional dose, toxicity and the local application of itraconazole to other tissues.

\section{Conclusions}

We report what is, to the best of our knowledge, the first case of fungal myositis successfully treated with intravenous and intra-lesional itraconazole in a patient with acute myelomonocytic leukemia. The efficacy and safety of the local administration of itraconazole for infected soft tissues requires further evaluation.

\section{Consent}

Written informed consent was obtained from the patient for publication of this case report and any accompanying images. A copy of the written consent is available for review by the Editor-in-Chief of this journal.

\section{Abbreviations \\ FLAG: Fludarabine and cytarabine and granulocyte-macrophage colony- stimulating factor; IFI: Invasive fungal infection; M4: Acute myelomonocytic leukemia.}

\section{Competing interests}

The authors declare that they have no competing interests.

\section{Authors' contributions}

$X$-JL reviewed our patient's clinical data, performed the literature search and wrote the initial draft. R-XY, M-QH, B-LZ and W-JG reviewed the initial draft and finalized the manuscript. All authors read and approved the final manuscript.

\section{Acknowledgements}

We acknowledge our patient for providing informed consent for this case report.

Received: 5 December 2012 Accepted: 17 April 2013

Published: 19 May 2013

\section{References}

1. Ascioglu S, Rex JH, De Pauw B: Defining opportunistic invasive fungal infections in immunocompromised patients with cancer and hematopoietic stem cell transplants: an international consensus. Clin Infect Dis 2002, 34:7-14.

2. Fornadley JA, Parker GS, Rickman LS, Paparello S: Candida myositis manifesting as a discrete neck mass. Otolaryngol Head Neck Surg 1990, 102:74-76.

3. Reboli AC, Reilly RF, Jacobson RJ: Aspergillus myositis in a patient with a myelodysplastic syndrome. Mycopathologia 1987, 97:117-119. 
4. Li DM, Xiu DR, Li RY, Samson RA, de Hoog GS, Wang DL: Aspergillus flavus myositis in a patient after liver transplantation. Clin Transplant 2008, 22:508-511.

5. Gave AA, Torres R, Kaplan L: Cryptococcal myositis and vasculitis: an unusual necrotizing soft tissue infection. Surg Infect 2004, 5:309-313.

6. Tsai SH, Peng YJ, Wang NC: Pyomyositis with hepatic and perinephric abscesses caused by Candida albicans in a diabetic nephropathy patient. Am J Med Sci 2006, 331:292-294.

7. Flynn PM, Marina NM, Rivera GK, Hughes WT: Candida tropicalis infections in children with leukemia. Leuk Lymphoma 1993, 10:369-376.

8. Schwartz DM, Morgan ER: Multimodality imaging of Candida tropicalis myositis. Pediatr Radiol 2008, 38:473-476.

9. Lee KS, Kim YH, Bae WK: Percutaneous intracavitary treatment of a giant aspergilloma (letter). AJR Am J Roentgenol 1990, 154:1346.

10. Shapiro MJ, Albelda SM, Mayock RL, McLean GK: Severe haemoptysis associated with pulmonary aspergilloma. Chest 1988, 96:1225-1231.

11. Hargis JL, Bone RC, Stewart J, Rector N, Huller FC: Intracavitary amphotencin-B in the treatment of symptomatic pulmonary aspergillomas. Am J Med 1980, 68:389-394.

12. Nouyrigat P, Baume D, Blaise D, Revillon D, Gabus R, Miquel M, Maraninchi D: Candida arthritis treated with intra-articular amphotericin B. Eur J Med 1993, 2:124-125.

13. Zmierczak H, Goemaere S, Mielants H, Verbruggen G, Veys EM: Candida glabrata arthritis: case report and review of the literature of Candida arthritis. Clin Rheumatol 1999, 18:406-409.

doi:10.1186/1752-1947-7-132

Cite this article as: Lin et al:: Treatment of fungal myositis with intra-

lesional and intravenous itraconazole: a case report. Journal of Medical Case Reports 2013 7:132.

\section{Submit your next manuscript to BioMed Central and take full advantage of:}

- Convenient online submission

- Thorough peer review

- No space constraints or color figure charges

- Immediate publication on acceptance

- Inclusion in PubMed, CAS, Scopus and Google Scholar

- Research which is freely available for redistribution 